\title{
Presarcopenia Is an Independent Risk Factor for Carotid Atherosclerosis in Chinese Population with Metabolic Syndrome
}

This article was published in the following Dove Press journal: Diabetes, Metabolic Syndrome and Obesity: Targets and Therapy

Yuan Cao,' Ming Zhong,' Yuke Zhang, Zijie Zheng,' Yapeng Liu,' Xiaoning $\mathrm{Ni},{ }^{\prime}$ Lu Han,, ,3 Ming Song,' Wei Zhang,' Zhihao Wang ${ }^{1,4}$

'The Key Laboratory of Cardiovascular Remodeling and Function Research, Chinese Ministry of Education, Chinese National Health Commission and Chinese Academy of Medical Sciences, The State and Shandong Province Joint Key Laboratory of Translational Cardiovascular Medicine, and The Department of Cardiology, Qilu Hospital of Shandong University, Jinan, Shandong, People's Republic of China; ${ }^{2}$ The First Affiliated Hospital of Shandong First Medical University, Shandong Provincial Qianfoshan Hospital, Department of Critical Care Medicine, Jinan, Shandong, People's Republic of China; ${ }^{3}$ Department of General Medicine, Qilu Hospital of Shandong University, Jinan, Shandong, People's Republic of China; ${ }^{4}$ Department of Geriatric Medicine, Qilu Hospital of Shandong University, and The Key Laboratory of Cardiovascular Proteomics of Shandong Province, Jinan, Shandong, People's Republic of China

\section{Correspondence: Wei Zhan}

The Key Laboratory of Cardiovascular Remodeling and Function Research, Chinese Ministry of

Education, Chinese National Health Commission and Chinese Academy of Medical Sciences, The State and Shandong Province Joint Key Laboratory of Translational Cardiovascular Medicine, and The Department of Cardiology, Qilu Hospital of

Shandong University, No. 107, Wen Hua Xi Road, Jinan 2500 I2, People's Republic of China

Tel +86 I85 60086586

Fax +86 53186169356

Email zhangweisdu7@।63.com

Zhihao Wang

The Key Laboratory of Cardiovascular Remodeling and Function Research, Chinese Ministry of Education, Chinese National Health Commission and Chinese Academy of Medical Sciences, The State and Shandong Province Joint Key Laboratory of Translational Cardiovascular Medicine, The

Department of Geriatric Medicine, Qilu Hospital of Shandong University, and The Key Laboratory of Cardiovascular Proteomics of Shandong Province,

No. 107, Wen Hua Xi Road, Jinan 2500 I2, People's

Republic of China

Tel +86 I85 60082268

Fax +8653186169356

Email zhihao369@163.com
Purpose: To investigate the impacts of skeletal muscle mass on carotid atherosclerosis in Chinese adults with metabolic syndrome (MetS).

Methods: One hundred and ninety-five subjects with MetS had the waist-to-height ratio (WHTR) $\geq 0.5$ for all. One hundred and eighty-four subjects without MetS were divided into 2 groups: Non-Mets obese group (WHTR $\geq 0.5, \mathrm{n}=118$ ) and Non-MetS control group (WHTR $<0.5, \mathrm{n}=66$ ). All the groups had no difference in age. Appendicular skeletal muscle mass was acquired and skeletal muscle mass index (SMI) was calculated. Carotid intima-media thickness (IMT) was assessed by ultrasonography. Each group was stratified according to the presence or absence of presarcopenia.

Results: While most parameters showed an increasing trend with WHTR and MetS in both genders, SMI and HDL-C showed a decreasing trend. The prevalence of carotid atherosclerosis showed the same increasing trend. Multivariate logistic regression analyses showed SBP and presarcopenia were both independent risk factors for carotid atherosclerosis in MetS (OR 1.026, $P<0.001$; OR 2.788, $P=0.001$, respectively). There was no significant difference in IMT among the three groups with preserved muscle mass whether the participants suffered from obesity or MetS, while there was a significant difference between the two groups with presarcopenia (in male $P=0.020$, in female $P=0.009$, respectively). The area under the ROC curve (AUC) was $0.641(P<0.001)$ for presarcopenia.

Conclusion: Obesity was a risk factor for sarcopenia independent of age, especially in subjects with metabolic syndrome. In individuals with MetS, our findings suggest that presarcopenia may be an independent risk factor for atherosclerosis and appendicular skeletal muscle mass had potential protective effects for carotid atherosclerosis regardless of gender. Keywords: skeletal muscle mass, presarcopenia, metabolic syndrome, carotid atherosclerosis

\section{Introduction}

Sarcopenia was first proposed in 1989 to describe age-related decrease of muscle mass and used to be recognized as a geriatric syndrome. ${ }^{1,2}$ In 2010, the European Working Group on Sarcopenia in Older People (EWGSOP, the Sarcopenia Working Group) gave a new definition and suggested a conceptual staging as presarcopenia characterized by low muscle mass without impact on muscle strength or physical performance. ${ }^{3}$ Recently, the concept of sarcopenia is changing: it is now recognized not as an inevitable outcome of ageing, but as a disease condition which could be overcome. ${ }^{4}$ Sarcopenia is more prevalent among people with obesity and exists 
with various comorbidities. ${ }^{3,4}$ Several cardiometabolic disorders, including diabetes mellitus, metabolic syndrome (MetS), and cardiovascular disease, have been associated with sarcopenia.

Obesity is reaching epidemic proportions with recent worldwide figures estimated at 1.4 billion and they are rising year by year. ${ }^{5}$ Obesity is now recognized as a chronic low-grade and systemic inflammatory state that predisposes to other chronic conditions including MetS. ${ }^{6}$ The MetS has been clearly demonstrated that the syndrome generally increases with advancing age and that it has a rising prevalence worldwide. ${ }^{7,8}$ The MetS and its constituent components have been reported to be risk factors for cardiovascular disease including atherosclerosis and increase cardiovascular morbidity and mortality. ${ }^{9,10}$ At present, insulin resistance, ${ }^{11}$ mitochondrial genome-encoded protein expression disorder, ${ }^{12}$ cross-talk of adipokines and myokines between adipose tissue, skeletal muscle and other organs, ${ }^{13}$ and inflammatory response ${ }^{6}$ may participate in the onset of a cardiovascular disease associated with MetS.

As one of the most important body compositions in metabolism, skeletal muscle is known to have a role in reversing the detrimental impact of MetS. ${ }^{13}$ Increased skeletal muscle mass can significantly reduce the risk of hypertension, dyslipidemia and type 2 diabetes. ${ }^{14}$ Recent studies have shown that sarcopenia and MetS usually coexist, ${ }^{15,16}$ which further increases the risk of cardiovascular disease in adults. ${ }^{17}$ Considering the concept that obesity was the common denominator between sarcopenia and MetS, a more robust investigation is necessary to determine the complex association of sarcopenia with MetS. Moreover, whether atherosclerosis associated with MetS is affected by skeletal muscle is still not fully understood.

\section{Materials and Methods Subjects}

Participants for this investigation $(n=394)$ aged $24-85$ years were recruited from the Qilu Hospital of Shandong University. One hundred and ninety-five subjects with MetS were defined by the International Diabetes Federation and the waist-to-height ratio (WHTR) $\geq 0.5$ for all. ${ }^{7,18}$ One hundred and eighty-four subjects without MetS were further divided into 2 groups according to WHTR: Non-MetS obese group (WHTR $\geq 0.5, \mathrm{n}=118$ ) and NonMetS control group (WHTR $<0.5, \mathrm{n}=66$ ). All the groups had no difference in age. All participants provided written informed consent, and the study protocol was approved by the medical ethics committee of Qilu Hospital of Shandong University. The ethics approval was given in compliance with the Declaration of Helsinki.

\section{Data Collection}

Anthropometric measurements were assessed. Standing height and body weight were measured according to the predetermined protocol. BMI was calculated as the body weight divided by the standing height squared $\left(\mathrm{kg} / \mathrm{m}^{2}\right)$. Waist circumference and hip circumference were assessed with waist-hip ratio calculated. Systolic blood pressure (SBP) and diastolic blood pressure (DBP) were measured, then the difference value as pulse pressure (PP).

Blood samples were collected from the antecubital vein after the patients had fasted for at least $8 \mathrm{hrs}$. Total cholesterol (TC), high-density lipoprotein cholesterol (HDL-C), low-density lipoprotein cholesterol (LDL-C), and triglyceride (TG) levels were measured. Fasting blood glucose and insulin concentrations, hemoglobin A1c (HbA1c) concentrations and uric acid (UA) were measured. HOMA-IR was calculated as multiply fasting blood glucose by insulin then divided by $22.5 .{ }^{19}$ HOMA-IR cut-off value was 2.5 to identify individuals with insulin resistance (IR).

\section{Appendicular Skeletal Muscle Mass Index}

ASM was acquired by anthropometric SM prediction formulas derived from MRI. ${ }^{20}$ Most of the studies referring to sarcopenia were conducted mainly through dual-energy $\mathrm{X}$-ray to obtain skeletal muscle mass, the main drawback of which is that the equipment is not portable and common for routine clinical practice, especially in ambulatory settings. $^{3,21}$ As we know, MRI is a gold standard for estimating muscle mass in research, ${ }^{3}$ so we consulted the literature and chosen an anthropometric prediction model acquired from MRI for an alternative:

$\operatorname{ASM}(\mathrm{kg})=0.244 \times \mathrm{BW}+7.80 \times \mathrm{Ht}-0.098 \times$ age $+6.6 \times$ sex + race-3.3, where sex $=1$ for male and 0 for female, race $=$ -1.2 for Asian, 1.4 for African American, and 0 for white or Hispanic.

The appendicular skeletal muscle mass index (ASMI) was calculated as follows: ASMI (\%) = total ASM $(\mathrm{kg}) /$ body weight $(\mathrm{kg}) \times 100$, which was modified from the study of Janssen et al. ${ }^{22,23}$ Presarcopenia was defined as 1 standard deviation (SD) below the sex-specific average value of skeletal muscle mass of the reference group. ${ }^{16,23}$ 


\section{Carotid Artery Ultrasonography}

Individuals underwent an ultrasound examination of the carotid artery according to the Asymptomatic Carotid Artery Plaque Study (ACAPS) protocol. ${ }^{24}$ The bilateral common carotid arteries were examined by certified sonographers. Subsequently, IMT of the common and internal carotid arteries and bifurcations were measured. Carotid atherosclerosis was defined as a maximal IMT $\geq 1.0 \mathrm{~mm} .{ }^{25-29}$ The measurements were made via $\mathrm{B}$ mode ultrasonography with a $5-10 \mathrm{MHz}$ linear probe (GE vivid 7, USA).

\section{Statistical Analysis}

Statistical analyses were performed using SPSS 20.0 (SPSS, Chicago, IL). Continuous data were presented as mean \pm standard deviation. We evaluated differences in demographic characteristics among the 6 groups based on the WHTR and MetS using one-way ANOVA analysis for normally distributed variables, the Kruskal-Wallis test for skewed variables, and the $\chi^{2}$ test for categorical variables. The association between metabolic disorders, presarcopenia and IMT was assessed binary logistic regression method. Multivariable logistic regression analyses models were used to assess the independent effect of SMI on the carotid atherosclerosis of MetS patients. Forward: LR selection method (with probability for entry being $<0.10$ ) and the backward: LR selection (with probability for entry and removal being 0.05 and 0.10 , respectively) procedures were performed sequentially to select and validate potential diagnostic variables. $P<0.05$ was considered significant. Variables with significant contributions to the IMT were plotted with the receiver-operating characteristic (ROC) curve.

\section{Results}

Baseline characteristics of all groups according to gender, WHTR and the presence of MetS were outlined in Table 1. For both males and females, the age had no differences among the three groups. In men, physical parameters (weight, BMI, waist, hip, WHR), metabolic parameters (cholesterol, LDL-C, triglyceride, UA, Glu, insulin, HbA1c, HOMA-IR) and carotid artery parameter (IMT) showed an increasing trend with WHTR and MetS. In women, physical parameters (SBP, DBP, PP, weight, BMI, waist, hip, WHR), metabolic parameters (cholesterol, LDL-C, triglyceride, UA, Glu, insulin, HbA1c, HOMA-IR) and carotid artery parameter (IMT) showed an increasing trend with WHTR and MetS. On the contrary, SMI and HDL-C showed a decreasing trend both in men and women.

All parameters that differed significantly among the MetS groups, obese groups and control groups were introduced into the binary logistic regression model for univariate analyses. SBP, DBP, PP, waist circumference, cholesterol, triglyceride, LDL-c, IR and presarcopenia were left in the regression model 1 (Table 2). These parameters were further used for multivariate analyses. After validation with forward: LR selection and backward: LR selection, SBP and presarcopenia were left in the regression model 2, the odds ratios of SBP and presarcopenia for detecting carotid atherosclerosis were 1.026 and 2.788 , respectively (Table 3 ).

According to the definition of presarcopenia and carotid atherosclerosis mentioned before, we stratified the study group to explore the relationship between presarcopenia and carotid atherosclerosis. As shown in Figure 1A, the prevalence of carotid atherosclerosis was significantly increased with increased WHTR and MetS in both genders. In female as shown in Figure 1B, there was significant difference in IMT between MetSpresarcopenia group and MetS-nonsarcopenia group $(P=0.009)$, MetS-presarcopenia group and obesenonsarcopenia group $(P<0.001)$, MetS-presarcopenia group and obese-presarcopenia group $(P=0.009)$, MetSpresarcopenia group and control group $(P<0.001)$. In male, as shown in Figure 1C, there was a significant difference in IMT between MetS-presarcopenia group and obese-presarcopenia group $(P=0.020)$, MetSpresarcopenia group and control group $(P=0.023)$. This result suggests that both metabolic syndrome components and muscle mass play an important role in carotid atherosclerosis. There was no significant difference in IMT among the three groups with preserved muscle mass whether the participants suffered from obesity or MetS (MetS-nonsarcopenia group, obese-nonsarcopenia group and control group), while there was significant difference between the two groups with presarcopenia (MetS-presarcopenia group and obese-presarcopenia group), indicating that muscle mass may have a certain protective effect.

The diagnostic value of MetS parameters and presarcopenia for carotid atherosclerosis is outlined in Figure 2. Based on above results, ROC curves were made in all patients with all risk parameters and the area under the ROC curve (AUC) was $0.641(P<0.001)$ for presarcopenia, $0.692(P<0.001)$ for SBP, $0.667(P<0.001)$ for DBP, $0.627(P=0.001)$ for waist 
Table I Demographic Characteristics and Clinical Parameters of the Study Population

\begin{tabular}{|c|c|c|c|c|c|c|}
\hline & \multicolumn{3}{|l|}{ Female } & \multicolumn{3}{|l|}{ Male } \\
\hline & $\begin{array}{l}\text { Non-MetS } \\
\text { Control Group } \\
(\mathrm{WHTR}<0.5)\end{array}$ & $\begin{array}{l}\text { Non-MetS } \\
\text { Obese Group } \\
(\text { WHTR } \geq 0.5)\end{array}$ & $\begin{array}{l}\text { MetS Group } \\
\text { (WHTR } \geq 0.5 \text { ) }\end{array}$ & $\begin{array}{l}\text { Non-MetS } \\
\text { Control Group } \\
\text { (WHTR<0.5) }\end{array}$ & $\begin{array}{l}\text { Non-MetS } \\
\text { Obese Group } \\
(\text { WHTR } \geq 0.5)\end{array}$ & $\begin{array}{l}\text { MetS Group } \\
\text { (WHTR } \geq 0.5)\end{array}$ \\
\hline $\mathrm{n}$ & 48 & 68 & 109 & 18 & 50 & $n=83$ \\
\hline Age, years & $53.26 .00 \pm 8.12$ & $53.76 \pm 9.00$ & $56.28 \pm 6.83$ & $50.50 \pm 7.88$ & $51.70 \pm 10.15$ & $49.90 \pm 9.66$ \\
\hline $\mathrm{SBP}, \mathrm{mmHg}$ & $111.27 \pm 10.59$ & || $6.97 \pm|0.4|$ & $\mid 51.38 \pm 21.42^{* \dagger}$ & $118.44 \pm 8.22$ & $115.90 \pm 9.63$ & $148.59 \pm 23.17^{* \dagger}$ \\
\hline $\mathrm{DBP}, \mathrm{mmHg}$ & $72.58 \pm 7.33$ & $75.01 \pm 6.18$ & $91.62 \pm 13.61^{* \dagger}$ & $76.33 \pm 7.93$ & $78.44 \pm 5.86$ & $97.42 \pm 13.36^{* \dagger}$ \\
\hline $\mathrm{PP}, \mathrm{mmHg}$ & $38.69 \pm 7.88$ & $41.96 \pm 8.89$ & $59.72 \pm 15.27^{* \dagger}$ & $42.11 \pm 4.27$ & $37.46 \pm 6.97$ & $51.23 \pm 16.30 * t$ \\
\hline Height, cm & $|60.08 \pm 5.0|$ & $158.12 \pm 4.23 *$ & I $58.75 \pm 4.89$ & $170.06 \pm 3.86$ & $169.76 \pm 3.82$ & $172.19 \pm 4.44^{\dagger}$ \\
\hline Weight, Kg & $56.61 \pm 6.62$ & $63.71 \pm 7.49 *$ & $72.14 \pm 9.71 * \dagger$ & $64.92 \pm 4.60$ & $75.47 \pm 8.19 *$ & $88.64 \pm 15.20 * \dagger$ \\
\hline BMI & $22.06 \pm 2.05$ & $25.45 \pm 2.58^{*}$ & $28.53 \pm 3.67 * t$ & $22.45 \pm 1.45$ & $26.16 \pm 2.43^{*}$ & $29.84 \pm 4.66 * \dagger$ \\
\hline $\begin{array}{l}\text { Waist } \\
\text { circumference, } \mathrm{cm}\end{array}$ & $74.17 \pm 4.13$ & $86.49 \pm 6.04 *$ & $94.72 \pm 9.20 * \dagger$ & $80.72 \pm 3.98$ & $91.96 \pm 4.97 *$ & $102.95 \pm 9.79 * \dagger$ \\
\hline $\begin{array}{l}\text { Hip } \\
\text { circumference, } \mathrm{cm}\end{array}$ & $93.50 \pm 6.07$ & $100.57 \pm 5.64 *$ & $105.13 \pm 8.09 * \dagger$ & $93.44 \pm 4.66$ & $101.86 \pm 5.46 *$ & $107.06 \pm 9.52 * t$ \\
\hline WHR & $0.79 \pm 0.04$ & $0.86 \pm 0.04 *$ & $0.90 \pm 0.06 * \dagger$ & $0.87 \pm 0.03$ & $0.90 \pm 0.04 *$ & $0.96 \pm 0.05 * \dagger$ \\
\hline $\begin{array}{l}\text { Cholesterol, } \\
\mathrm{mmol} / \mathrm{L}\end{array}$ & $4.43 \pm 0.72$ & $4.74 \pm 0.88$ & $5.54 \pm 1.12 * \dagger$ & $4.29 \pm 1.01$ & $4.63 \pm 0.61$ & $5.20 \pm 1.08 * \dagger$ \\
\hline $\mathrm{HDL}-\mathrm{C}, \mathrm{mmol} / \mathrm{L}$ & $\mathrm{I} .60 \pm 0.34$ & $1.57 \pm 0.33$ & $1.33 \pm 0.38^{* t}$ & $1.52 \pm 0.39$ & $\mathrm{I} .4 \mathrm{I} \pm 0.28$ & $1.10 \pm 0.23 * t$ \\
\hline LDL-C, mmol/L & $2.69 \pm 0.67$ & $2.99 \pm 0.74$ & $3.72 \pm 1.00 * t$ & $2.57 \pm 0.83$ & $3.03 \pm 0.57$ & $3.42 \pm 0.92 * \dagger$ \\
\hline $\begin{array}{l}\text { Triglyceride, } \\
\mathrm{mmol} / \mathrm{L}\end{array}$ & $0.92 \pm 0.31$ & $1.11 \pm 0.50$ & $2.16 \pm 1.41^{* \dagger}$ & $0.95 \pm 0.44$ & $1.13 \pm 0.37$ & $2.45 \pm 1.12^{* \dagger}$ \\
\hline $\mathrm{UA}, \mu \mathrm{mol} / \mathrm{L}$ & $222.38 \pm 45.67$ & $234.69 \pm 72.64$ & $294.21 \pm 69.88 * \dagger$ & $301.24 \pm 97.37$ & $309.00 \pm 59.12$ & $371.22 \pm 84.14^{* \dagger}$ \\
\hline Glu, mmol/L & $4.70 \pm 0.40$ & $4.92 \pm 0.63$ & $6.79 \pm 2.84 * \dagger$ & $4.7 I \pm 0.69$ & $5.02 \pm 0.55$ & $6.42 \pm 2.01 * \dagger$ \\
\hline Insulin, $\mu \mathrm{U} / \mathrm{mL}$ & $9.7 I \pm 3.50$ & II. $.79 \pm 5.34$ & $19.23 \pm 8.19 * \dagger$ & $7.27 \pm 1.36$ & $11.26 \pm 5.03$ & $21.89 \pm 12.97 * \dagger$ \\
\hline HbAlc, \% & $4.53 \pm 0.20$ & $4.7 I \pm 0.37$ & $5.56 \pm 1.37^{* \dagger}$ & $4.50 \pm 0.29$ & $4.65 \pm 0.30$ & $5.30 \pm 0.94 * \dagger$ \\
\hline HOMA-IR & $2.01 \pm 0.89$ & $2.65 \pm 1.38$ & $5.81 \pm 3.65 * \dagger$ & $1.54 \pm 0.40$ & $2.55 \pm 1.31$ & $6.27 \pm 4.60 * \dagger$ \\
\hline IMT, mm & $0.84 \pm 0.94$ & $0.8 I \pm 0.46$ & $1.18 \pm 0.8 I^{* \dagger}$ & $0.68 \pm 0.71$ & $0.81 \pm 0.75$ & $1.20 \pm 0.90 * \dagger$ \\
\hline ASMI, \% & $30.27 \pm 1.84$ & $28.41 \pm 1.59 *$ & $27.78 \pm 1.44 * \dagger$ & $40.5 \mathrm{I} \pm \mathrm{I} .47$ & $38.14 \pm 1.81 *$ & $36.62 \pm 1.63 * t$ \\
\hline
\end{tabular}

Notes: In female, ${ }^{*} P<0.05$ compared with Non-MetS control group (WHTR $\left.<0.5\right) ;{ }^{\dagger} P<0.05$ compared with Non-MetS obese group $($ WHTR $\geq 0.5)$. In male, ${ }^{*} P<0.05$ compared with Non-MetS control group (WHTR<0.5); ${ }^{\dagger} P<0.05$ compared with Non-MetS obese group (WHTR $\geq 0.5$ ).

Abbreviations: MetS, metabolic syndrome; SBP, systolic blood pressure; DBP, diastolic blood pressure; PP, pulse pressure; BMI, body mass index; WHR, waist-hip ratio; HDL-C, high-density lipoprotein cholesterol; LDL-C, low-density lipoprotein cholesterol; UA, uric acid; Glu, glucose; HbA lc, hemoglobin AI c; IMT, intima-media thickness; ASMI, appendicular skeletal muscle mass.

circumference, $0.610(P=0.005)$ for TC, $0.614(P=0.003)$ for LDL-C, $0.610(P=0.005)$ for triglyceride, 0.569 $(P=0.079)$ for insulin resistance, respectively.

\section{Discussion}

This population-based study demonstrated that obesity was a risk factor for muscle mass loss independent of age, especially in subjects with metabolic syndrome. Furthermore, in subjects with simple obesity, the reduction in muscle mass did not affect carotid atherosclerosis, and the reduction in muscle mass in the case of metabolic syndrome promoted carotid atherosclerosis. Muscle content had potential protective effects on carotid atherosclerosis regardless of gender.

WHTR, as a simple screening tool, was reliable as BMI and waist circumference in identifying metabolic and vascular differences between populations. ${ }^{30}$ In a recent study, WHTR appeared to be the best indicator of dyslipidemia, hyperglycemia, and CVDs. ${ }^{18}$ So in the present study, we used this parameter as a cutoff of control group and obese group. Consistent with the previous finding, ${ }^{4}$ accelerated muscle loss occurs in people with diabetes and obesity. The present study also found that as the severity of disease increased, from simple obesity to metabolic syndrome, SMI was gradually decreasing, and the incidence of carotid atherosclerosis was gradually increasing.

The MetS are highly associated with increased morbidity and mortality of atherosclerotic cardiovascular disease (ASCVD). Large-scale population studies have shown that obesity, elevated triglycerides, and LDL-C, hypertension, and hyperglycemia in metabolic syndrome 
Table 2 Multivariate Logistic Regression Analyses Model I in Patients with MetS and Healthy Controls

\begin{tabular}{|l|l|l|l|l|l|l|}
\hline Factors & $\boldsymbol{\beta}$ & SE & Wals & P & OR & 95\% CI \\
\hline SBP, $\mathrm{mmHg}$ & 0.024 & 0.005 & 16.947 & 0.000 & 1.024 & $1.014-1.035$ \\
$\mathrm{DBP}, \mathrm{mmHg}$ & 0.030 & 0.009 & 12.224 & 0.000 & 1.031 & $1.013-1.048$ \\
$\mathrm{PP}, \mathrm{mmHg}$ & 0.028 & 0.008 & 12.214 & 0.000 & 1.029 & $1.013-1.045$ \\
Waist circumference, cm & 0.034 & 0.011 & 10.270 & 0.001 & 1.035 & $1.013-1.056$ \\
Cholesterol, mmol/L & $0.31 \mathrm{I}$ & 0.119 & 6.862 & 0.009 & 1.365 & $1.081-1.723$ \\
Triglyceride, mmol/L & 0.237 & 0.116 & 4.198 & 0.040 & 1.268 & $1.010-1.591$ \\
LDL-C, mmol/L & 0.385 & 0.138 & 7.777 & 0.005 & 1.470 & $1.121-1.927$ \\
IR & 0.604 & 0.293 & 4.233 & 0.040 & 1.829 & $1.029-3.251$ \\
Presarcopenia & 1.140 & 0.266 & 18.342 & 0.000 & 3.126 & $1.855-5.266$ \\
\hline
\end{tabular}

Abbreviations: $\mathrm{Cl}$, confidence interval; OR, odds ratio; SE, standard error; SBP, systolic blood pressure; DBP, diastolic blood pressure; PP, pulse pressure; LDL-C, lowdensity lipoprotein cholesterol; IR, insulin resistance.

Table 3 Multivariate Logistic Regression Analyses Model 2 in Patients with MetS and Healthy Controls

\begin{tabular}{|l|l|l|l|l|l|l|}
\hline Factors & $\boldsymbol{\beta}$ & SE & Wals & $\mathbf{P}$ & OR & $\mathbf{9 5 \%} \mathbf{C l}$ \\
\hline SBP, mmHg & 0.021 & 0.006 & 13.549 & $<0.001$ & 1.026 & $1.010-1.033$ \\
Presarcopenia & 1.025 & 0.296 & 11.960 & 0.001 & 2.788 & $1.559-4.985$ \\
Constant & -4.497 & 0.807 & 31.040 & $<0.001$ & 0.011 & \\
\hline
\end{tabular}

Abbreviations: $\mathrm{Cl}$, confidence interval; OR, odds ratio; SE, standard error; SBP, systolic blood pressure.

all contribute to coronary atherosclerosis. ${ }^{9}$ Although the pathogenic processes of developing presarcopenia have not been fully understood, presarcopenia and MetS may share common pathophysiologic mechanisms. ${ }^{6}$ Increased insulin resistance, which is a major causative factor of MetS, ${ }^{31}$ can also aggravate presarcopenia via mitochondrial dysfunction ${ }^{32}$ and the degradation of muscle protein by the activation of the ubiquitin-proteasome proteolytic pathway. ${ }^{33,34}$ Additionally, insulin resistance is the key link between metabolic syndrome and ASCVD. ${ }^{31,35,36}$
Sanada et $\mathrm{al}^{17}$ have shown that the coexistence of sarcopenia and MetS in adult Japanese women significantly increases the risks of cardiovascular disease. Studies have also shown that skeletal muscle mass can affect carotid intima thickness ${ }^{37,38}$ and is closely related to the occurrence of subclinical atherosclerosis. ${ }^{39-42}$ The present study suggested that both metabolic syndrome components and muscle mass loss play an important role in carotid atherosclerosis. Binary logistic regression analysis showed that SBP and presarcopenia were both
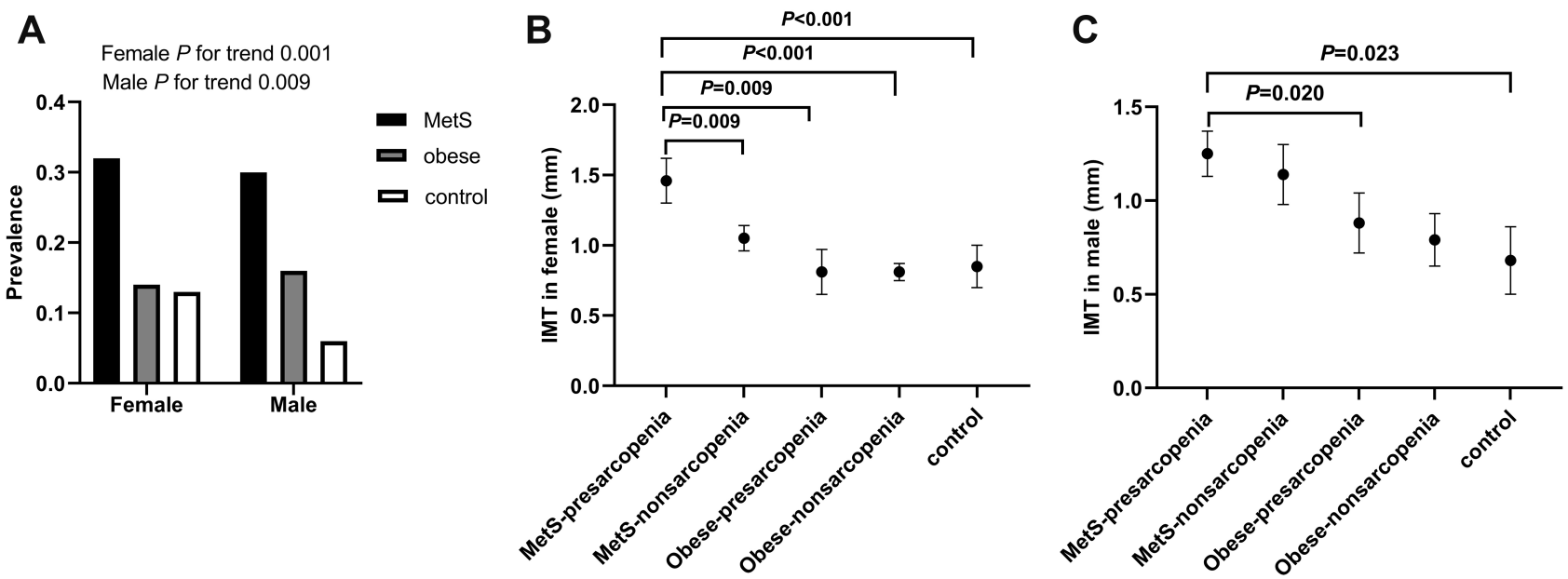

Figure I (A) Comparison of the prevalence of carotid atherosclerosis in both genders. (B) and (C) Comparison of IMT among the study groups stratified according to ASMI in both genders.

Abbreviations: MetS, metabolic syndrome; IMT, intima-media thickness. 


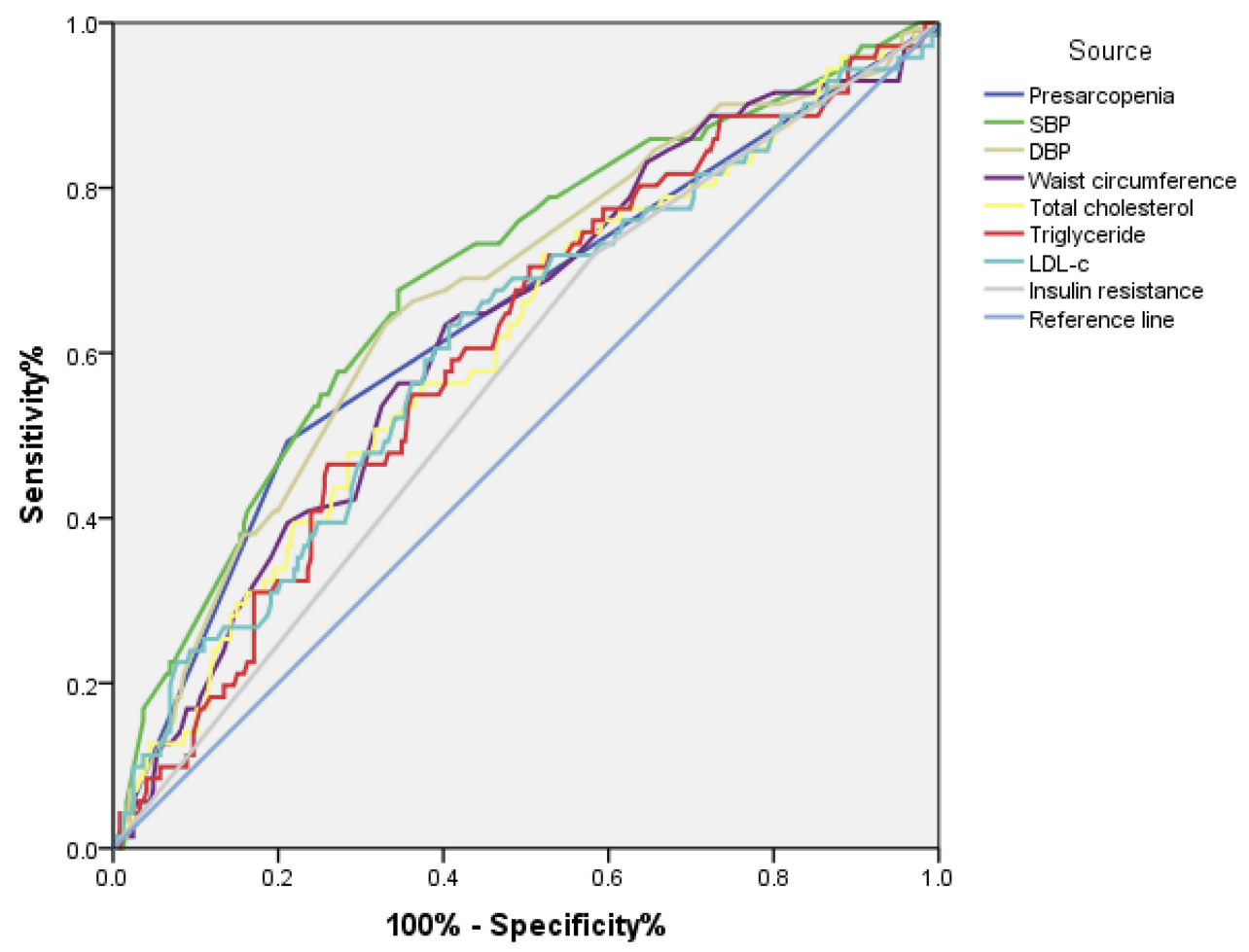

Figure 2 Receiver operating characteristic (ROC) curve analysis of different parameters for predicting carotid atherosclerosis. The area under the curve (AUC) was 0.64 I for presarcopenia $(P<0.00 \mathrm{I}), 0.692$ for SBP $(P<0.00 \mathrm{I}), 0.667$ for DBP $(P<0.00 \mathrm{I}), 0.627$ for waist circumference $(P=0.00 \mathrm{I}), 0.6 \mathrm{I} 0$ for TC $(P=0.005), 0.6 \mathrm{I} 4$ for LDL-C $(P=$ $0.003), 0.610$ for triglyceride $(P=0.005), 0.569$ for insulin resistance $(P=0.079)$, respectively.

Abbreviations: SBP, systolic blood pressure; DBP, diastolic blood pressure; LDL-C, low-density lipoprotein cholesterol.

independent risk factors for carotid atherosclerosis. The AUC for presarcopenia confirmed it.

According to our findings, muscle mass declines with advancing obese and metabolic disorders, and that this liaison favors the obesity-related and metabolism-related acceleration of atherogenesis. ${ }^{43}$ Obesity and sarcopenia influenced each other, which may lead to a vicious cycle. The reduction in physical activity due to sarcopenia induced decreased energy expenditure and increased the possibility of obesity. As visceral obesity increased, catabolic inflammatory responses were upregulated and contributed to reduced muscle mass. ${ }^{13}$ Moreover, our study examined the differential effects of presarcopenia and metabolic disorders on the risks of carotid atherosclerosis. That is, despite the gender, compared with individuals with preserved skeletal muscle mass, subjects with presarcopenia had a higher risk of carotid atherosclerosis independent of the status of obesity as well as metabolic syndrome.

Sarcopenia lacks early diagnostic tools and debate is continuing about how best to define and measure sarcopenia. These results were encouraging. Firstly, it was verified that the protective effect of muscle mass in the development of atherosclerosis and would encourage people to pay attention to the function of skeletal muscle mass in the early stage of the disease. Secondly, it could promote the development of a simple mobile phone application to monitor muscle mass anytime and anywhere.

Our study had some limitations that warrant discussion. First of all, this study stems from a cross-sectional study, which allows no conclusion on cause and effect. Secondly, the sample size of this study is relatively small, and it has certain limitations in the wide-scale promotion of the research results. Thirdly, the determination of the muscle mass of the subjects in this study is based on the anthropometric formula calculation method, instead of the dualenergy X-ray, bioelectrical impedance and other objective methods by means of instruments and equipment. Because the formula applies to a different population, there may be some deviations between the calculation results and the real values. Ideally, using an objective method such as dual-energy X-ray to carry out a follow-up study in a larger population may help to further clarify the relationship between muscle mass and metabolism syndrome atherosclerosis.

In conclusion, obesity was a risk factor for muscle mass loss independent of age, especially in subjects with 
metabolic syndrome. Furthermore, despite the gender, subjects with presarcopenia had a higher risk of carotid atherosclerosis independent of the status of obesity as well as metabolic syndrome compared with individuals with preserved skeletal muscle mass. Muscle content had potential protective effects on carotid atherosclerosis regardless of gender.

\section{Acknowledgments}

This work was supported by the research grants from the National Natural Science Foundation of China (81873534, 81702194, 81600633, 81670411, 81570400, 81801953, 81470560, 81471036), Key Research and Development Program of Shandong Province (2018GSF118002, 2018GSF118017, 2017GSF18156), the Natural Science Foundation of Shandong Province (ZR2014HQ037, ZR2017BH023).

\section{Disclosure}

The authors report no conflicts of interest in this work.

\section{References}

1. Rosenberg IH. Sarcopenia: origins and clinical relevance. $J$ Nutr. 1997;127(5 Suppl):990S-991S. doi:10.1093/jn/127.5.990S

2. Epidemiologic and methodologic problems in determining nutritional status of older persons. Proceedings of a conference. Albuquerque, New Mexico, October 19-21, 1988. Am J Clin Nutr. 1989;50(5 Suppl):1121-1235.

3. Cruz-Jentoft AJ, Baeyens JP, Bauer JM, et al. Sarcopenia: European consensus on definition and diagnosis: report of the European working group on sarcopenia in older people. Age Ageing. 2010;39 (4):412-423. doi:10.1093/ageing/afq034

4. Lancet Diabetes \& Endocrinology, T. Sarcopenia: a fate worth challenging. Lancet Diabetes Endocrinol. 2014;2(3):183. doi:10.10 16/S2213-8587(14)70055-5

5. O'Neill S, O'Driscoll L. Metabolic syndrome: a closer look at the growing epidemic and its associated pathologies. Obes Rev. 2015;16 (1):1-12. doi:10.1111/obr.12229

6. Collins KH, Herzog W, MacDonald GZ, et al. Obesity, metabolic syndrome, and musculoskeletal disease: common inflammatory pathways suggest a central role for loss of muscle integrity. Front Physiol. 2018;9:112. doi:10.3389/fphys.2018.00112

7. Alberti KG, Zimmet P, Shaw J. The metabolic syndrome-a new worldwide definition. Lancet. 2005;366(9491):1059-1062. doi:10.10 16/S0140-6736(05)67402-8

8. Ford ES. Prevalence of the metabolic syndrome defined by the international diabetes federation among adults in the U.S. Diabetes Care. 2005;28(11):2745-2749. doi:10.2337/diacare.28.11.2745

9. Bayturan O, Tuzcu EM, Lavoie A, et al. The metabolic syndrome, its component risk factors, and progression of coronary atherosclerosis. Arch Intern Med. 2010;170(5):478-484. doi:10.1001/archinternmed.2009.551

10. Klein S, Burke LE, Bray GA, et al. Clinical implications of obesity with specific focus on cardiovascular disease: a statement for professionals from the American Heart Association Council on Nutrition, Physical Activity, and Metabolism: endorsed by the American College of Cardiology Foundation. Circulation. 2004;110 (18):2952-2967. doi:10.1161/01.CIR.0000145546.97738.1E
11. Oda E. Metabolic syndrome: its history, mechanisms, and limitations. Acta Diabetol. 2012;49(2):89-95. doi:10.1007/s00592-011-0309-6

12. Zheng N, Wei D, Dai B, et al. Mitochondrial genome encoded proteins expression disorder, the possible mechanism of the heart disease in metabolic syndrome. Cell Physiol Biochem. 2017;43 (3):959-968. doi:10.1159/000481649

13. Chung HS, Choi KM. Adipokines and myokines: a pivotal role in metabolic and cardiovascular disorders. Curr Med Chem. 2018;25 (20):2401-2415. doi:10.2174/0929867325666171205144627

14. Kim TN, Choi KM. The implications of sarcopenia and sarcopenic obesity on cardiometabolic disease. J Cell Biochem. 2015;116 (7):1171-1178. doi:10.1002/jcb.v116.7

15. Buchmann N, Nikolov J, Spira D, et al. Identifying sarcopenia in metabolic syndrome: data from the berlin aging study II. $J$ Gerontol a Biol Sci Med Sci. 2016;71(2):265-272. doi:10.1093/gerona/glv089

16. Lim S, Kim JH, Yoon JW, et al. Sarcopenic obesity: prevalence and association with metabolic syndrome in the Korean Longitudinal Study on Health and Aging (KLoSHA). Diabetes Care. 2010;33 (7):1652-1654. doi:10.2337/dc10-0107

17. Sanada K, Iemitsu M, Murakami H, et al. Adverse effects of coexistence of sarcopenia and metabolic syndrome in Japanese women. Eur J Clin Nutr. 2012;66(10):1093-1098. doi:10.1038/ejcn.2012.43

18. Liu J, Tse LA, Liu Z, et al. Predictive values of anthropometric measurements for cardiometabolic risk factors and cardiovascular diseases among 44048 Chinese. J Am Heart Assoc. 2019;8(16): e010870. doi:10.1161/JAHA.118.010870

19. Katz A, Nambi SS, Mather K, et al. Quantitative insulin sensitivity check index: a simple, accurate method for assessing insulin sensitivity in humans. J Clin Endocrinol Metab. 2000;85(7):2402-2410. doi:10.1210/jcem.85.7.6661

20. Lee RC, Wang Z, Heo M, Ross R, Janssen I, Heymsfield SB. Totalbody skeletal muscle mass: development and cross-validation of anthropometric prediction models. Am J Clin Nutr. 2000;72 (3):796-803. doi:10.1093/ajcn/72.3.796

21. Chen LK, Liu LK, Woo J, et al. Sarcopenia in Asia: consensus report of the Asian working group for sarcopenia. J Am Med Dir Assoc. 2014;15(2):95-101. doi:10.1016/j.jamda.2013.11.025

22. Lee YH, Jung KS, Kim SU, et al. Sarcopaenia is associated with NAFLD independently of obesity and insulin resistance: nationwide surveys (KNHANES 2008-2011). J Hepatol. 2015;63(2):486-493. doi:10.1016/j.jhep.2015.02.051

23. Janssen I, Heymsfield SB, Ross R. Low relative skeletal muscle mass (sarcopenia) in older persons is associated with functional impairment and physical disability. $J$ Am Geriatr Soc. 2002;50(5):889-896. doi:10.1046/j.1532-5415.2002.50216.x

24. Espeland MA, Craven TE, Riley WA, Corson J, Romont A, Furberg CD. Reliability of longitudinal ultrasonographic measurements of carotid intimal-medial thicknesses. Asymptomatic carotid artery progression study research group. Stroke. 1996;27(3):480-485. doi:10.1161/01.STR.27.3.480

25. Stein JH, Korcarz CE, Hurst RT, et al. Use of carotid ultrasound to identify subclinical vascular disease and evaluate cardiovascular disease risk: a consensus statement from the American society of echocardiography carotid intima-media thickness task force. Endorsed by the society for vascular medicine. J Am Soc Echocardiogr. 2008;21 (2):93-111; quiz 189-190. doi:10.1016/j.echo.2007.11.011

26. Bots ML, Hoes AW, Koudstaal PJ, Hofman A, Grobbee DE. Common carotid intima-media thickness and risk of stroke and myocardial infarction: the rotterdam study. Circulation. 1997;96 (5):1432-1437. doi:10.1161/01.CIR.96.5.1432

27. Naqvi TZ, Lee MS. Carotid intima-media thickness and plaque in cardiovascular risk assessment. JACC Cardiovasc Imaging. 2014;7 (10):1025-1038. doi:10.1016/j.jcmg.2013.11.014

28. Gu X, Fang X, Hua Y, et al. Association between kidney dysfunction and carotid atherosclerosis in community-based older adults in China. Angiology. 2016;67(3):252-258. doi:10.1177/0003319715586505 
29. Johnson HM, Stein JH. Measurement of carotid intima-media thickness and carotid plaque detection for cardiovascular risk assessment. J Nucl Cardiol. 2011;18(1):153-162. doi:10.1007/s12 350-010-9319-y

30. Maher V, O’Dowd M, Carey M, et al. Association of central obesity with early carotid intima-media thickening is independent of that from other risk factors. Int $J$ Obes (Lond). 2009;33(1):136-143. doi:10.1038/ijo.2008.254

31. Reaven GM. Insulin resistance: the link between obesity and cardiovascular disease. Endocrinol Metab Clin North Am. 2008;37(3):581-601, vii-viii. doi:10.1016/j.ecl.2008.06.005

32. Johnson ML, Robinson MM, Nair KS. Skeletal muscle aging and the mitochondrion. Trends Endocrinol Metab. 2013;24(5):247-256. doi:10.1016/j.tem.2012.12.003

33. Cleasby ME, Jamieson PM, Atherton PJ. Insulin resistance and sarcopenia: mechanistic links between common co-morbidities. J Endocrinol. 2016;229(2):R67-R81. doi:10.1530/JOE-15-0533

34. Kalyani RR, Corriere M, Ferrucci L. Age-related and disease-related muscle loss: the effect of diabetes, obesity, and other diseases. Lancet Diabetes Endocrinol. 2014;2(10):819-829. doi:10.1016/S2213-85 87(14)70034-8

35. Reddy KJ, Singh M, Bangit JR, Batsell RR. The role of insulin resistance in the pathogenesis of atherosclerotic cardiovascular disease: an updated review. J Cardiovasc Med (Hagerstown). 2010;11 (9):633-647. doi:10.2459/JCM.0b013e328333645a

36. Ormazabal V, Nair S, Elfeky O, Aguayo C, Salomon C, Zuniga FA. Association between insulin resistance and the development of cardiovascular disease. Cardiovasc Diabetol. 2018;17(1):122. doi:10.11 86/s12933-018-0762-4
37. Heo JE, Kim HC, Shim JS, et al. Association of appendicular skeletal muscle mass with carotid intima-media thickness according to body mass index in Korean adults. Epidemiol Health. 2018;40:e2018049. doi:10.4178/epih.e2018049

38. Hida T, Imagama S, Ando K, et al. Sarcopenia and physical function are associated with inflammation and arteriosclerosis in community-dwelling people: the Yakumo study. Mod Rheumatol. 2018;28(2):345-350. doi:10.1080/14397595.2017.1349058

39. Abbatecola AM, Chiodini P, Gallo C, et al. Pulse wave velocity is associated with muscle mass decline: health ABC study. Age (Dordr). 2012;34(2):469-478. doi:10.1007/s11357-011-9238-0

40. Campos AM, Moura FA, Santos SN, Freitas WM, Sposito AC. Sarcopenia, but not excess weight or increased caloric intake, is associated with coronary subclinical atherosclerosis in the very elderly. Atherosclerosis. 2017;258:138-144. doi:10.1016/j.atherosclerosis.2017. 01.005

41. Kato A, Ishida J, Endo Y, et al. Association of abdominal visceral adiposity and thigh sarcopenia with changes of arteriosclerosis in haemodialysis patients. Nephrol Dial Transplant. 2011;26(6):19 67-1976. doi:10.1093/ndt/gfq652

42. Ochi M, Kohara K, Tabara Y, et al. Arterial stiffness is associated with low thigh muscle mass in middle-aged to elderly men. Atherosclerosis. 2010;212(1):327-332. doi:10.1016/j.atherosclerosis.2010.05.026

43. Yang EJ, Lim S, Lim JY, Kim KW, Jang HC, Paik NJ. Association between muscle strength and metabolic syndrome in older Korean men and women: the Korean longitudinal study on health and aging. Metabolism. 2012;61(3):317-324. doi:10.1016/j.metabol.2011.07.005

\section{Publish your work in this journal}

Diabetes, Metabolic Syndrome and Obesity: Targets and Therapy is an international, peer-reviewed open-access journal committed to the rapid publication of the latest laboratory and clinical findings in the fields of diabetes, metabolic syndrome and obesity research. Original research, review, case reports, hypothesis formation, expert opinion and commentaries are all considered for publication. The manuscript management system is completely online and includes a very quick and fair peer-review system, which is all easy to use. Visit http://www.dovepress.com/testimonials.php to read real quotes from published authors.

Submit your manuscript here: https://www.dovepress.com/diabetes-metabolic-syndrome-and-obesity-targets-and-therapy-journal 